\title{
The Prognostic Impact of Susceptibility-Weighted Imaging Prominent Veins in Acute Ischemic Stroke: A Systematic Review and Meta-Analysis
}

Ping Lu'

Lingyun Cui (D)

Xingquan Zhao $\mathbb{D}^{1-3}$

'Department of Neurology, Beijing Tiantan Hospital, Capital Medical University, Beijing, People's Republic of China; ${ }^{2}$ China National Clinical Research Center for Neurological Diseases, Capital Medical University, Beijing, People's Republic of China; ${ }^{3}$ Research Unit of Artificial Intelligence in Cerebrovascular Disease, Chinese Academy of Medical Sciences, Beijing, People's Republic of China
Correspondence: Xingquan Zhao Department of Neurology, Beijing Tiantan Hospital, Capital Medical University, Chinese Academy of Medical Sciences, I I9\#Nan Si Huan Xi Lu, Beijing, 100070, People's Republic of China

Tel +86 I80-102I-2600

Email zxq@vip.163.com
Purpose: We aimed to determine the prognostic impact of prominent veins (PVS) after an acute ischemic stroke identified on susceptibility-weighted imaging (PVS-SWI).

Methods: We searched for studies published in PubMed, Embase, Cochrane Library and Chinese Biomedical Literature Database. Poor functional prognosis, early neurological deterioration, and hemorrhagic transformation were evaluated. Risk ratios (RR) were pooled implementing a random effect model. We performed a subgroup analysis by treatment, location (cortical/medullary) and a sensitivity analysis by follow-up time.

Results: Sixteen studies were included (a total of 1605 patients) in the quantitative metaanalysis. PVS-SWI were related with a poor functional outcome (RR 1.62, 95\% CI 1.25 to 2.10), especially in the patients receiving thrombolysis (RR 2.19,95\% CI 1.53 to 3.15 ) and an augmented risk of early neurological damage (RR 2.85, 95\% CI 2.31 to 3.51). Both cortical and medullary prominent veins were accompanied by a poor functional outcome (RR $1.82,95 \%$ CI 1.30 to $2.56 /$ RR $2.59,95 \%$ CI 1.98 to 3.38 ). PVS-SWI were not associated with poor functional outcomes when patients were treated conservatively (RR $1.35,95 \% \mathrm{CI}$ 0.82 to 2.22 ), or with an increased risk of hemorrhagic transformation (RR $0.97,95 \% \mathrm{CI}$ 0.64 to 1.47 ).

Conclusion: PVS-SWI were related to a poor functional prognosis and an increased risk of early neurological damage. In patients treated conservatively, PVS-SWI were not accompanied by a poor prognosis. PVS-SWI were not associated with an augmented risk of hemorrhagic transformation.

Keywords: SWI, prominent veins, stroke, ischemic stroke, meta-analysis

\section{Introduction}

In the last three decades, stroke mortality has increased dramatically. ${ }^{1-3}$ The most common stroke subtype is acute ischemic stroke, accounting for $87 \%$ of all strokes. ${ }^{4}$ Early prognostic assessment aids the selection and implementation of interventions. Susceptibility-weighted imaging (SWI) is a useful magnetic resonance imaging (MRI) technique that allows a clearer visualization of the ischemic brain. $^{5,6}$ A prominent vessel sign (PVS) is one of the most pertinent aspects, representing the abnormally dilated cortical and medullary veins in the hypoperfusion tissue. Around $81 \%$ of patients who were investigated by SWI show prominent veins. $^{7}$ Although prominent veins on SWI correlate with the amount of deoxyhemoglobin in venous blood ${ }^{8}$ and venous dilatation, ${ }^{9}$ indirectly reflecting an increased oxygen extraction fraction (OEF) in brain tissues, its prognostic significance has not 
been clarified and remains controversial. Of the 16 studies which have so far investigated the prognosis of patients with PVS on SWI (PVS-SWI), 11 studies concluded that PVS-SWI-positive patients had a worse prognosis than PVS-SWI-negative patients. ${ }^{10-20}$ The remaining 5 studies found no differences between the two groups. ${ }^{21-25}$ Potential reasons for the different results in previous studies include small sample sizes, different treatment approaches, and diverse PVS-SWI definitions or assessment methods.

We hypothesized that PVS-SWI may be associated with poor prognosis in patients with ischemic stroke. We conducted a comprehensive evaluation to study the relationship between PVS-SWI and functional prognosis, early neurological deterioration, and hemorrhagic transformation in acute ischemic stroke.

\section{Methods}

The protocol followed the Preferred Reporting Items for Systematic Evaluation and Meta-Analysis (PRISMA) guidelines $^{26}$ and has been registered with PROSPERO (CRD42021244358).

\section{Search Strategy}

Our search strategy was conducted across relevant databases (PubMed, Embase, Cochrane Library and Chinese Biomedical Literature Database) based on the following combinations of terms or keywords for medical subject terms: ["hypointense" OR "prominent" OR "asymmetric*" OR "cortical" OR "medullary" OR "deep cerebral"] AND ["SWI" OR "susceptibility-weighted"] AND ["cerebrovascular disease" OR "stroke" OR "cerebral infarction"] (Supplementary Appendix 1). We searched from inception to March 31, 2021 without language restrictions. In addition, we manually searched articles in the reference list of all retrieved studies and previous reviews.

\section{Eligibility Criteria}

We included cohort studies conducted in patients $\geq 18$ years of age. A baseline MRI was performed in the acute stroke phase, with the time window varying up to 1 week. Eligible studies reported prognostic outcomes for patients with and without PVS-SWI. We excluded: editorials, letters, reviews, case series, conference abstracts, guidelines, technical notes, and book chapters. If studies overlapped, we only included the study with the largest number of patients.

\section{Study Selection and Data Extraction}

The titles and abstracts of identified studies were screened by two authors (LP and CL) and eligible studies were included in our analysis. Quality of such studies was assessed with the Newcastle-Ottawa Scale (NOS), with Level A $(\geq 6)$ or Level B $(<6)$. Data were extracted for pre-determined outcome measures using a standardized form. The data extraction form was categorized into subsections: (a) study characteristics (b) demographic, clinical, and imaging features of patients with and without PVS-SWI (c) outcome definitions (d) outcome metrics (see below). Any disagreement was resolved by discussion or negotiation.

The extracted data include:

(1) First author name, year and type of publication, type of study, inclusion criteria, study population, clinical and imaging features of recruited patients; (2) study treatment administered (thrombolysis, conservative therapy, or a combination of them); (3) There are four methods of extracted data in literatures: Visual assessment method, which classified patients as with PVS if hypointense vessels were observed in the ipsilateral hemisphere compared to the contralateral side. Patients were graded with the Alberta Stroke Program Early CT Score (ASPECTS), in which a quantitative score was utilized to classify patients into the SWI-PVS-positive group. The slice assessment method $(\geq 5$ slice planes defined a SWI-PVSpositive patient on 10 consecutive slices containing the MCA region) and the pixel comparison method (SWI-PVSpositive patients were classified through the difference in venous signal intensity of bilateral cerebral hemispheres); (4) primary outcome and other outcomes in patients with and without PVS-SWI on baseline MRI.

In the absence of data, we contacted the authors identified in the article.

\section{Outcomes}

The primary outcome was the functional prognosis evaluated with the modified Rankin Scale (mRS) or the National Institutes of Health Stroke Scale (NIHSS). The $\mathrm{mRS}$ is an ordinal scale that ranges from 0 (no symptoms) to 6 (death). Other outcomes included early neurological deterioration, and hemorrhagic transformation.

\section{Statistical Analysis}

The combined risk ratio (RR) for dichotomous outcomes was estimated using the DerSimonian-Laird random-effect model. Heterogeneity was evaluated using $\mathrm{I}^{2}$ statistics and 
Labbè plots. Subgroup analyses of primary outcomes were conducted according to treatment of patients, and type of PVS-SWI defined by location. Our meta-analysis explored whether clinical and radiological findings influenced the appearance of baseline PVS-SWI, expressed as RR. Funnel plots were visually examined to assess potential publication bias. A two-sided $p$-value $\leq 0.05$ was considered statistically significant. Statistical analysis was performed using the Stata software (v.16.0).

\section{Results}

\section{Study Selection and Characteristics}

The literature search provided a total of 461 articles (Figure 1). After titles and abstracts screening, 38 studies were reviewed in-full. Ten studies were conference abstracts, 11 studies did not meet the eligibility criteria. Two studies had overlapping cohort populations, therefore only the study with the largest population was included in the systematic review. ${ }^{19,27}$ Sixteen cohort studies met the inclusion criteria, ${ }^{10-25}$ involving 1605 patients. The included studies were published between 2011 and 2019, with stenosis and infarction of the larger cerebral arteries in the anterior circulation as the predominant lesions. Fourteen studies involving 1356 patients assessed functional outcomes, ${ }^{10-17,21-25,27}$ eleven of them (1216 patients) reported the outcome at 90 days. $^{10-15,21-24,27}$ Three studies performed thrombolysis $(\mathrm{n}=230) .{ }^{13,15,19}$ Five studies $(\mathrm{n}=251)$ reported the risk of hemorrhagic transformation, ${ }^{13,16,24,25,27}$ while 4 studies $(\mathrm{n}=875)$ reported early neurological deterioration. $^{10,18-20}$ Thirteen studies $(\mathrm{n}=1338)$ reported baseline NIHSS scores at admission. ${ }^{10,11,13-16,20-25,27}$ Six studies $(n=365)$ reported infarct volume at admission. ${ }^{14-16,21-23}$ Data from 13 studies $(n=1399)$ were pooled for predicting the prognostic impact of PVS-SWI. ${ }^{10,11,13-16,20-25,27}$ Two out of 16 studies were of low quality, with a high risk of bias (Table 1, Appendix Table 1). ${ }^{18,25}$

\section{Association of PVS-SWI with Functional Prognosis}

Severe stenosis/occlusion of the larger cerebral arteries was significantly associated with PVS-SWI (RR 2.51,

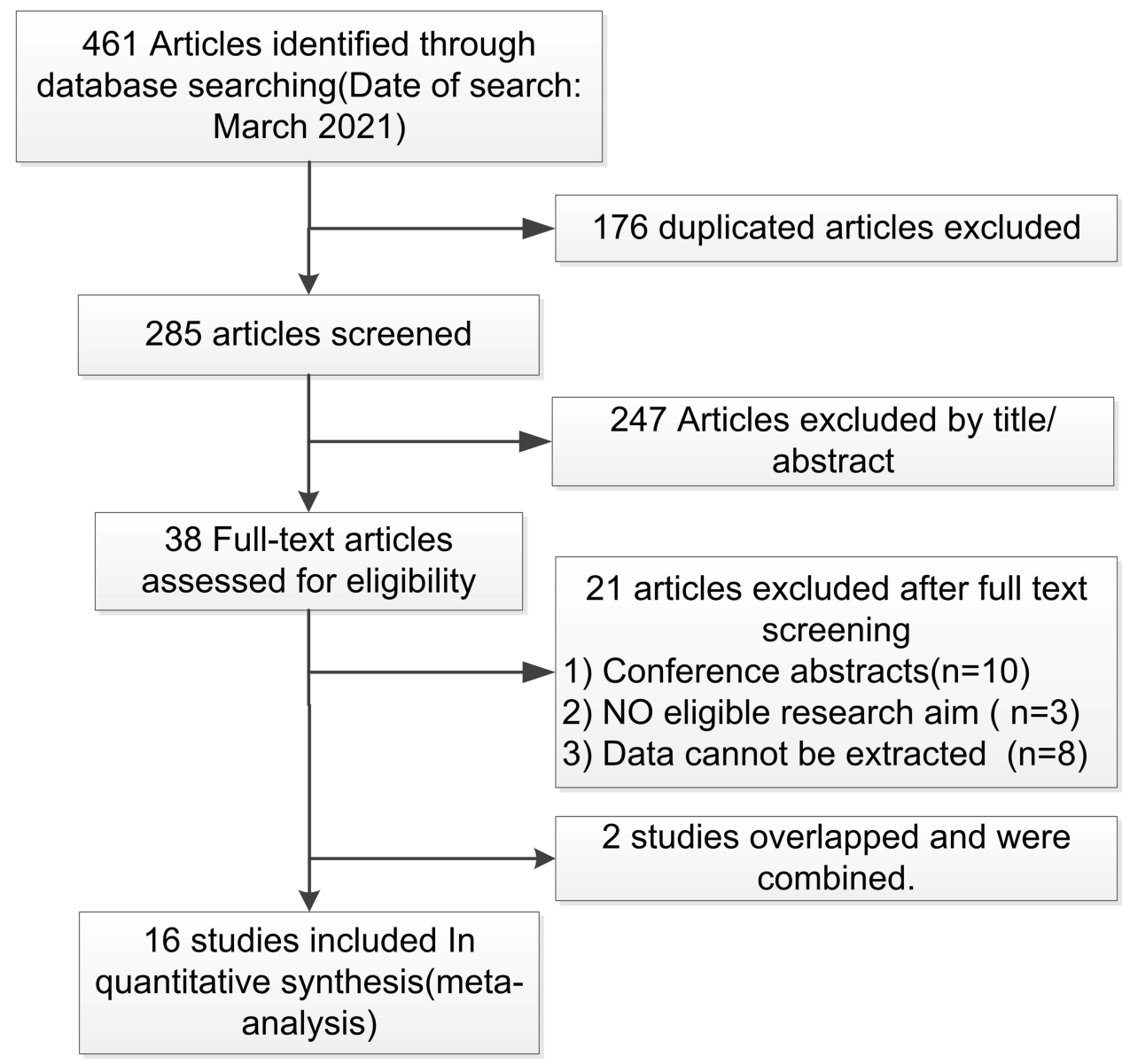

Figure I Selection process of the studies included in the review. 


\begin{tabular}{|c|c|c|c|c|c|c|c|c|c|c|c|c|c|c|c|c|c|c|}
\hline ֻ̆ & $\infty$ & 0 & n & 0 & $\infty$ & $\wedge$ & 0 & 0 & $m$ & $\infty$ & & $m$ & 0 & 0 & $\infty$ & $\wedge$ & $\infty$ & 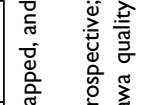 \\
\hline 1 & ' & ' & ' & ' & $\star$ & $*$ & $*$ & ' & & ' & & ' & 旸 & $*$ & 计 & ' & ' & 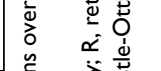 \\
\hline$\underline{n}$ & $*$ & $*$ & ' & $*$ & $\star$ & $*$ & * & ' & & 计 & & 计 & 计 & $*$ & $*$ & $*$ & $*$ & 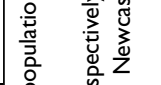 \\
\hline 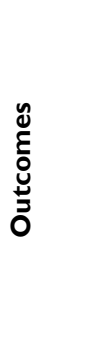 & 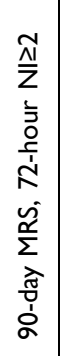 & 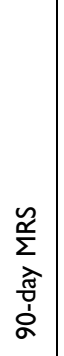 & 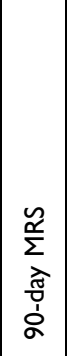 & 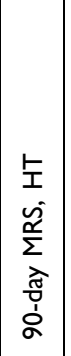 & 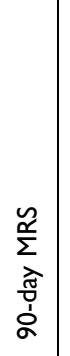 & 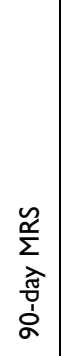 & 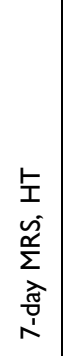 & 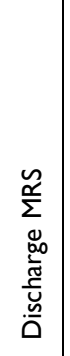 & 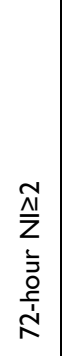 & 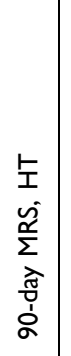 & 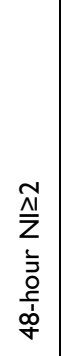 & 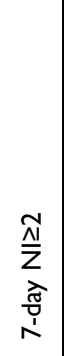 & 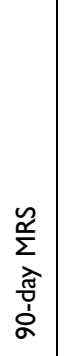 & 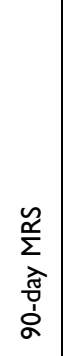 & 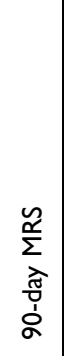 & 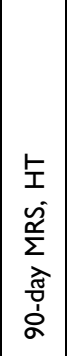 & 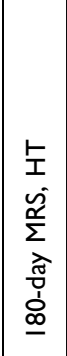 & 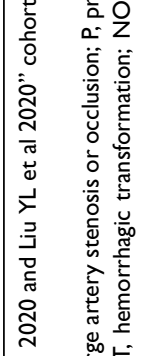 \\
\hline $\bar{\varangle}$ & ลิ & $\stackrel{ \pm}{\beth}$ & 우 & 8 & $\stackrel{m}{m}$ & ô & $\hat{f}$ & in & $\stackrel{\circ}{\circ}$ & นึก & $\overline{0}$ & $\stackrel{m}{\underline{m}}$ & ஓ & $\approx$ & $\stackrel{\sim}{\simeq}$ & $\mathscr{f}$ & $\Varangle$ & \\
\hline 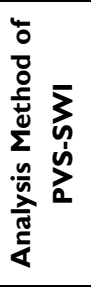 & 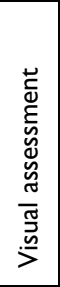 & 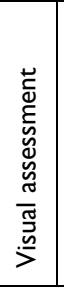 & 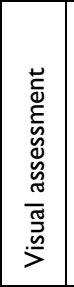 & 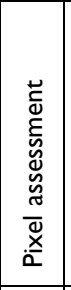 & 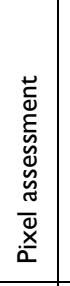 & 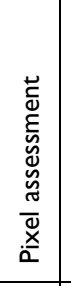 & 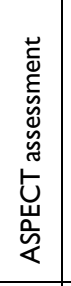 & 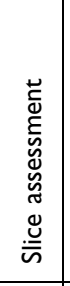 & 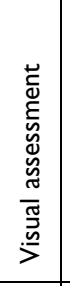 & 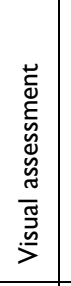 & 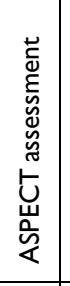 & 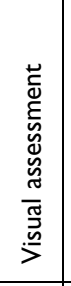 & 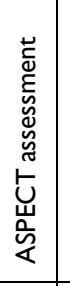 & 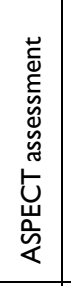 & 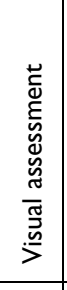 & 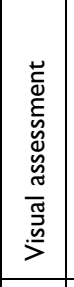 & 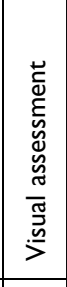 & 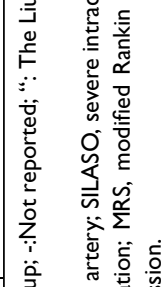 \\
\hline 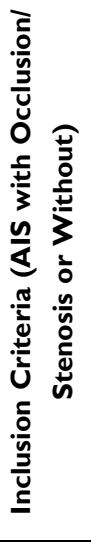 & $\mid \begin{array}{l}0 \\
0 \\
\vdots \\
\overline{5} \\
5 \\
0 \\
0 \\
\frac{1}{5} \\
3\end{array}$ & 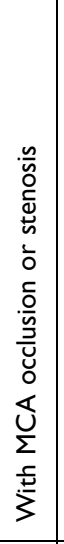 & 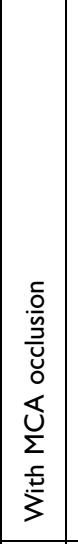 & 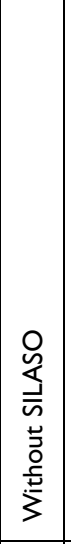 & 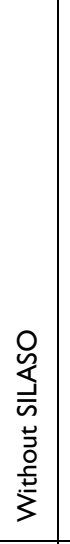 & 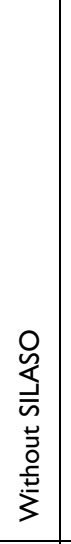 & 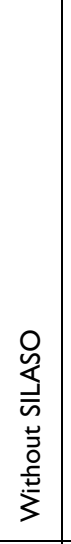 & 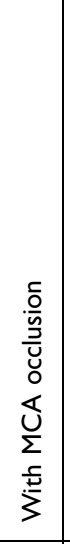 & 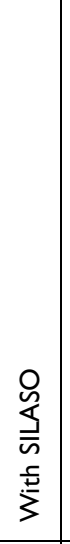 & $\begin{array}{l}0 \\
\dot{w} \\
\overline{5} \\
\frac{1}{5} \\
\sum\end{array}$ & & 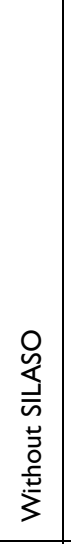 & 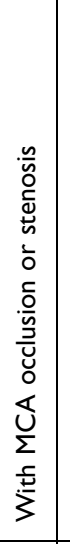 & 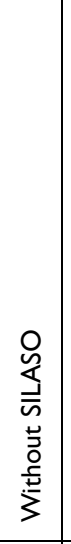 & 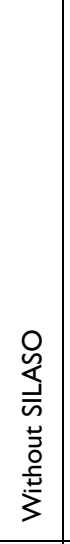 & 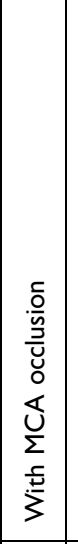 & 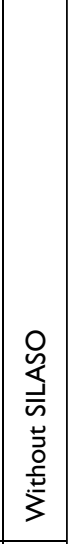 & 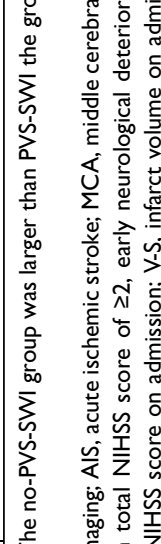 \\
\hline 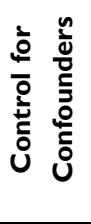 & 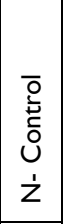 & 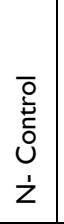 & 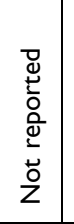 & $\begin{array}{l}\overline{0} \\
\text { ờ } \\
\text { Oे }\end{array}$ & $\begin{array}{l}\overline{0} \\
\stackrel{0}{2} \\
0 \\
0 \\
z \\
z\end{array}$ & 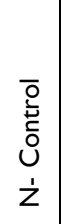 & $\begin{array}{l}\overline{0} \\
\overline{0} \\
\bar{u} \\
\dot{z} \\
z\end{array}$ & 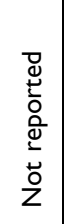 & 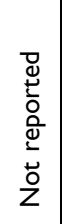 & $\begin{array}{l}\overline{0} \\
\stackrel{\bar{t}}{\grave{0}} \\
\text { uे }\end{array}$ & & 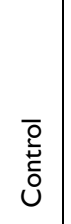 & $\begin{array}{l}\overline{0} \\
\overline{0} \\
\overline{0} \\
\dot{U} \\
\dot{z}\end{array}$ & 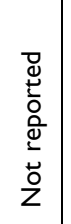 & $\begin{array}{l}\overline{0} \\
\dot{0} \\
0 \\
\dot{0} \\
\dot{z}\end{array}$ & 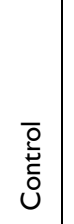 & 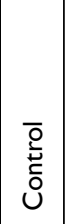 & 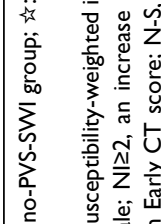 \\
\hline פू. & a & $\simeq$ & $\propto$ & $\propto$ & $a$ & $\propto$ & $\propto$ & $\simeq$ & $\propto$ & a & & $\simeq$ & $\propto$ & a & $a$ & $\simeq$ & a & 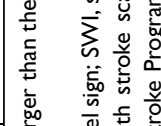 \\
\hline 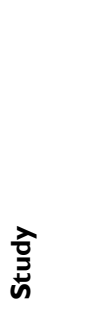 & 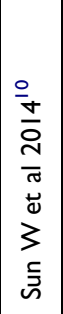 & 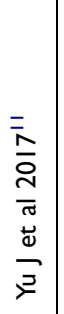 & 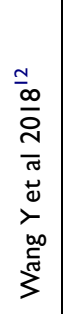 & 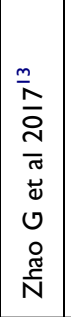 & 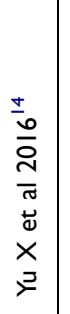 & 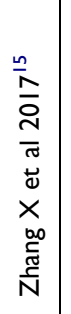 & 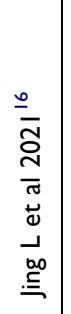 & 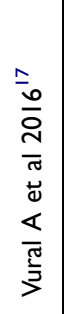 & 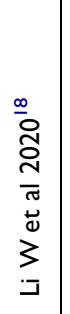 & 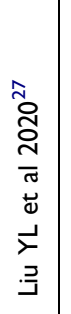 & 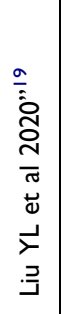 & 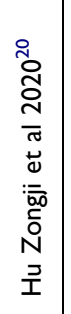 & 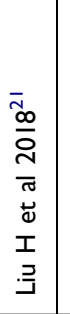 & 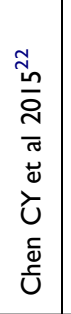 & 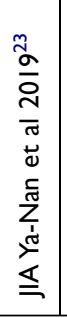 & 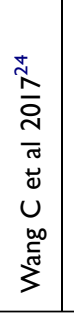 & 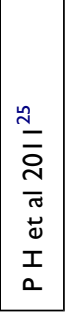 & 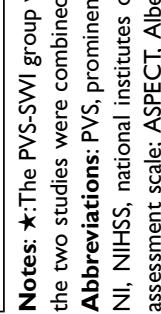 \\
\hline
\end{tabular}


Table 2 Meta-Analysis of the Associations of Baseline Factors with the Presence of PVS-SWI

\begin{tabular}{|c|c|c|c|c|}
\hline Factors & $\begin{array}{l}\text { Studies } \\
\text { (Patients) }\end{array}$ & $\begin{array}{l}\text { Risk Ratio or Mean Difference } \\
\qquad(95 \% \mathrm{Cl})\end{array}$ & $\begin{array}{l}\text { Heterogeneity } \\
\quad 12(\%)\end{array}$ & $\begin{array}{l}\text { Reference Number of } \\
\text { Included Studies }\end{array}$ \\
\hline $\begin{array}{l}\text { Age (with PVS-SWI vs } \\
\text { without, year) }\end{array}$ & $12(1266)$ & $-1.48[-3.48,0.5 \mid]$ & 20 & {$[10,11,13-16,21-25,27]$} \\
\hline Male vs female & $13(1400)$ & $1.06[0.93,1.21]$ & 0 & {$[10,11,13-16,20-25,27]$} \\
\hline Smoking vs no & $7(1087)$ & $1.00[0.8 \mathrm{I}, 1.24]$ & 18 & {$[10,13-15,20,23,27]$} \\
\hline $\begin{array}{l}\text { With prior stroke/TIA vs } \\
\text { without }\end{array}$ & $5(482)$ & $1.20[0.94,1.54]$ & 24 & {$[13,15,20,23,27]$} \\
\hline $\begin{array}{l}\text { With organic heart disease vs } \\
\text { without }\end{array}$ & $4(890)$ & $1.02[0.78,1.34]$ & 0 & {$[10,13,20,23]$} \\
\hline With AF vs without & $8(1224)$ & $1.12[0.95,1.31]$ & 0 & {$[10,11,13,15,20,23,24,27]$} \\
\hline With dyslipidemia vs without & $6(969)$ & $1.19[0.89,1.59]$ & 34 & {$[10,13,14,20,23,24]$} \\
\hline With DM vs without & $10(|30|)$ & $0.96[0.83,1.1 \mathrm{I}]$ & 0 & {$[10,11,13-15,20,23-25,27]$} \\
\hline With hypertension vs without & $10(|30|)$ & $0.96[0.78,1.17]$ & 41 & {$[10,11, \mid 13-15,20,23-25,27]$} \\
\hline With SILASO vs without & $7(1143)$ & $2.5 \mathrm{I}[\mathrm{I} .48,4.26]$ & 77 & {$[10,11,14-16,20,23]$} \\
\hline
\end{tabular}

Abbreviations: PVS, prominent vessel sign; SWI, susceptibility-weighted imaging; AF, atrial fibrillation; DM, diabetes mellitus; TIA, transient ischemic attack; SILASO, severe intracranial large artery stenosis or occlusion.

95\% CI 1.48 to $4.26 ; \mathrm{p}<0.001 \mathrm{I}^{2}=77 \%$ )(Table 2). Overall, PVS-SWI were related to a poorer prognosis (RR 1.62, $95 \%$ CI 1.25 to $2.10 ; \mathrm{p}<0.001 \mathrm{I}^{2}=70.41 \%$ )(Figure 2 ). In patients treated with thrombolytic therapy, a significant association between a poor prognosis and PVS-SWI was observed (RR 2.19, 95\% CI 1.53 to 3.15; $p<0.001 \mathrm{I}^{2}=0 \%$ ), but not in patients treated with conservative therapy (RR $1.35,95 \%$ CI 0.82 to $2.22 ; \mathrm{p}=0.12 \mathrm{I}^{2}=72.43 \%$ ). Similar results were observed with the 90-day functional outcome (RR 1.78, 95\% CI 1.34 to $2.78 ; \mathrm{p}=0.04 \mathrm{I}^{2}=66.81 \%$ ) (Appendix Figure 1). In subgroup analysis, corticomedullary differentiation correlated with a poor functional prognosis (RR 1.82, 95\% CI 1.30 to 2.56 ; $p<0.001 \mathrm{I}^{2}=68.65 \%$ / RR 2.59, 95\% CI 1.98 to 3.38; p $<0.001 \quad \mathrm{I}^{2}=0 \%$ ) (Figure 3A).

\section{Association of PVS-SWI with Other Outcomes}

PVS-SWI were associated with early neurological deterioration (RR 2.85, 95\% CI 2.31 to $3.51 ; \mathrm{p}<0.001 \mathrm{I}^{2}=0 \%$ ) but not with an increased risk of hemorrhagic transformation ( $\mathrm{RR} 0.97,95 \% \mathrm{CI} 0.64$ to $1.47 ; \mathrm{p}=0.89 \mathrm{I}^{2}=0 \%$ ) (Figure 3B). The funnel plot showed that there was no publication bias for the meta-analysis' parameters (Appendix Figure 2). The Labbè plot showed high heterogeneity for overall and 90-days functional prognosis parameters, but low heterogeneity for early neurological deterioration and hemorrhagic transformation parameters (Appendix Figure 3). Patients with PVS-SWI had higher baseline NIHSS scores and a greater initial and subsequent growth of infarct size than controls.

\section{Discussion}

We aimed to identify the prognostic impact of PVS-SWI in ischemic stroke. We found that the appearance of PVSSWI after AIS was associated with severe stenosis or occlusion of large cerebral arteries. Overall, PVS-SWI were related to a poor prognosis in patients treated with thrombolysis, but not in patients treated conservatively. PVS-SWI were also associated with early neurological deterioration, but not with an increased risk of hemorrhagic transformation.

\section{PVS Formation and Influencing Factors}

There is a consensus about the mechanism of PVS formation. Overall, PVS-SWI are associated with oxygen demand of brain tissue and the dilation of blood vessels. In order to maintain a normal oxygen metabolism, the oxygen extraction fraction (OEF) is increased during an ischemic stroke. The amount of deoxyhemoglobin in the venous blood determines the venous visualization on 


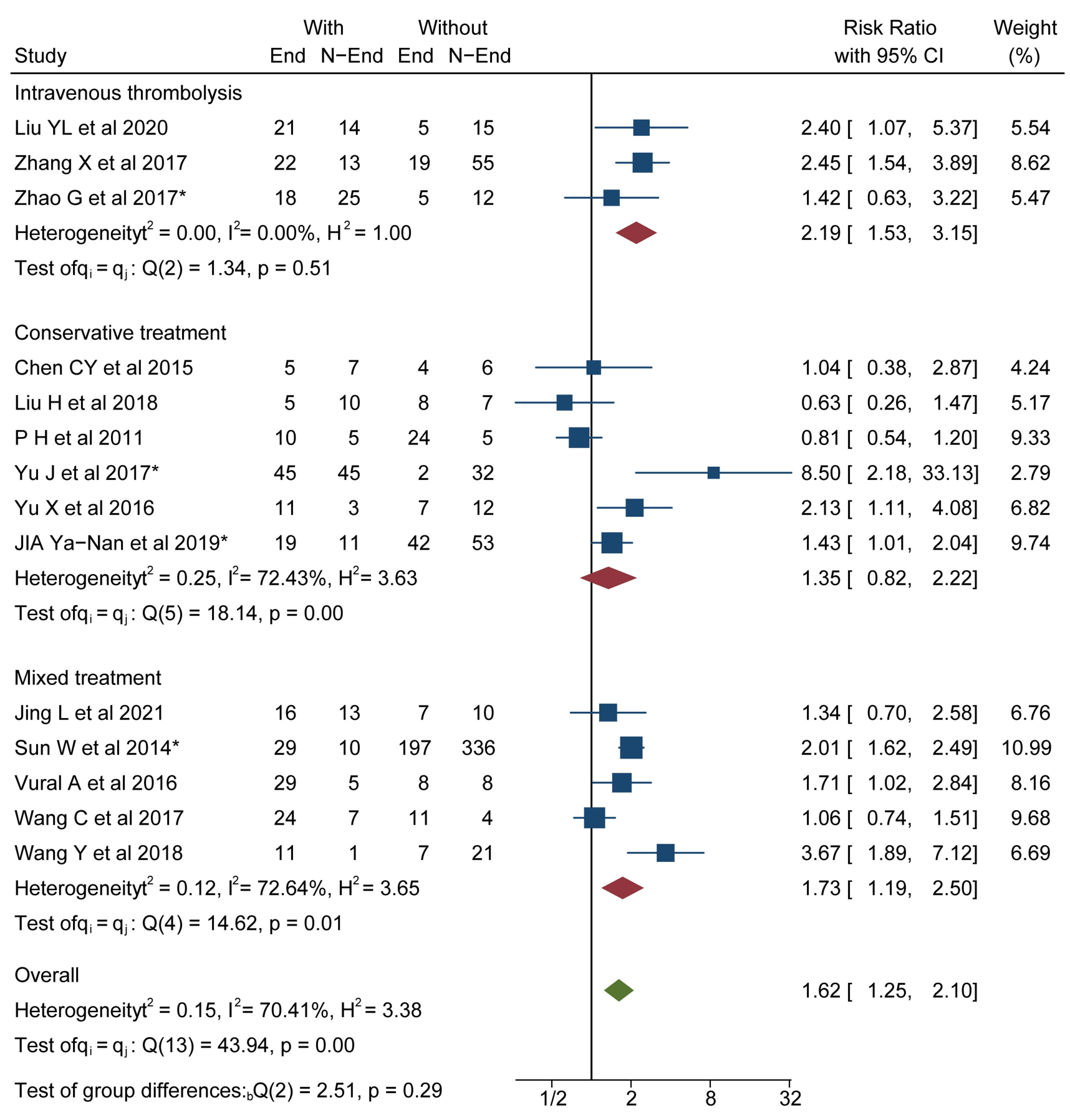

Figure 2 Meta-analysis of associations between modality of treatment and functional prognosis.

Abbreviations: PVS, prominent vessel sign; SWI, susceptibility-weighted imaging; End, unfavorable functional outcome; N-End, without unfavorable functional outcome; *mRS, scores 2-6 was regarded as unfavorable functional outcome.

SWI. ${ }^{5,8}$ When the delivery of oxygen falls, the amount of oxygen extracted from the blood increases to meet the metabolic demands of ischemic tissues, leading to the increase of deoxygenated hemoglobin in the venous blood. ${ }^{28}$ At the same time, the slow flow in the ischemic area further increases the deoxygenated hemoglobin concentration, ${ }^{29}$ resulting in a characteristic low signal intensity on SWI. The compensatory dilation of small arteries and resistance vessels maintains a relatively constant blood flow, increasing venous blood flow and enhancing veins dilation. ${ }^{9,25}$ In acute ischemic stroke, a severe stenosis of the larger cerebral arteries predisposes to a massive hypoperfusion of brain tissues, ${ }^{11,30}$ resulting in dilated veins and an increased deoxyhemoglobin/ 


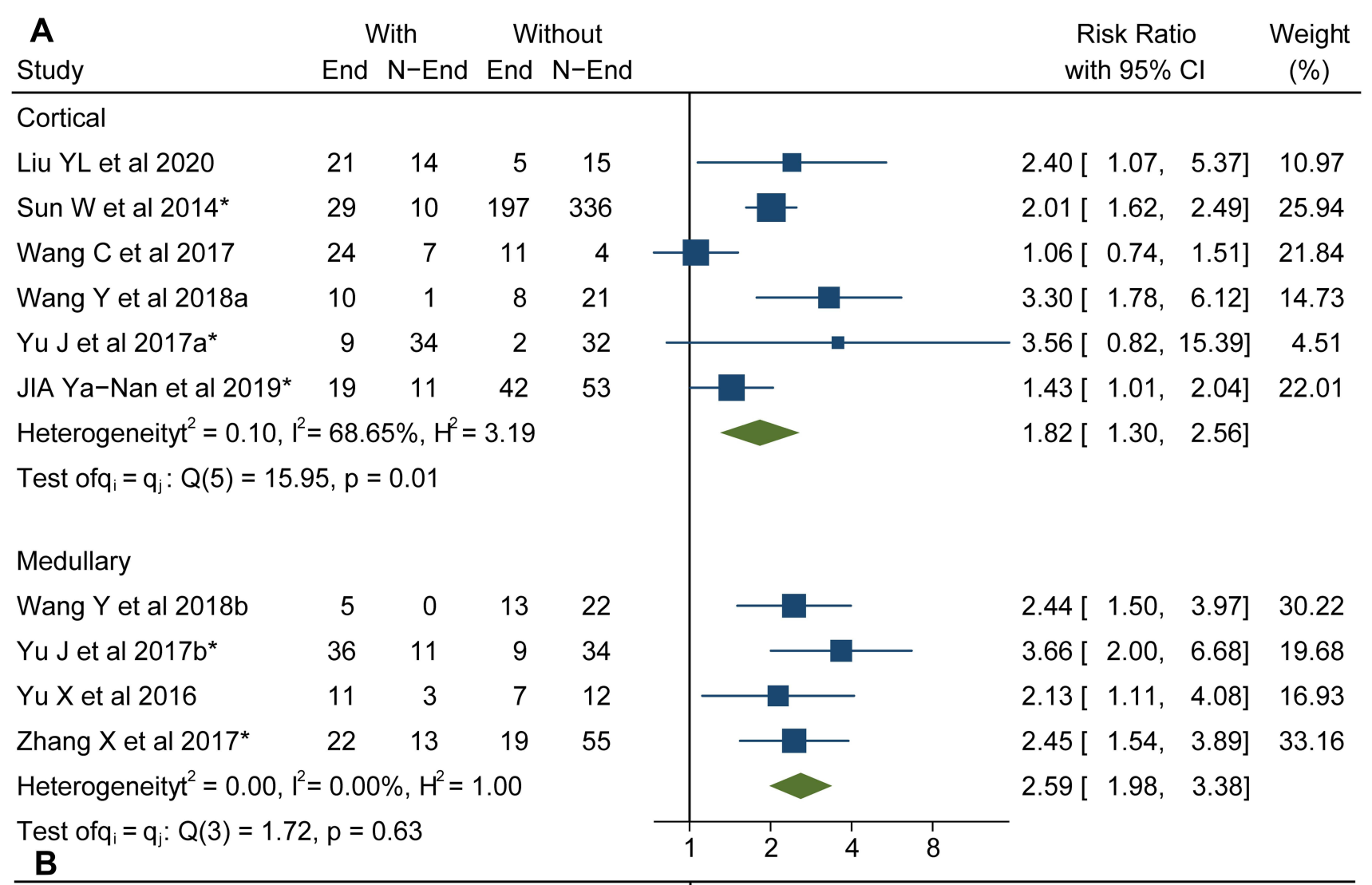

\section{NIHSS}

Liu YL et al 2020

Sun W et al 2014

$\begin{array}{rrrr}14 & 13 & 6 & 28 \\ 28 & 11 & 141 & 392\end{array}$

Hu Zongji et al 2020

$\begin{array}{llll}26 & 25 & 12 & 70\end{array}$

Li W et al 2020

$\begin{array}{llll}24 & 36 & 6 & 43\end{array}$

Heterogeneityt ${ }^{2}=0.00, I^{2}=0.00 \%, H^{2}=1.00$

Test ofq $q_{i}=q_{j}: Q(3)=0.72, p=0.87$

hemorrhagic transformation

Liu YL et al 2020*

Zhao G et al 2017

Wang $C$ et al 2017

Jing $L$ et al 2021

$\mathrm{P}$ H et al 2011

$\begin{array}{rrr}13 & 22 & 5 \\ 2 & 41 & 0 \\ 13 & 18 & 7 \\ 7 & 23 & 3 \\ 5 & 10 & 15\end{array}$

Heterogeneityt $^{2}=0.00, I^{2}=0.00 \%, H^{2}=1.00$

Test ofq $q_{i}=q_{j}: Q(4)=2.38, p=0.67$

28
392
70
43

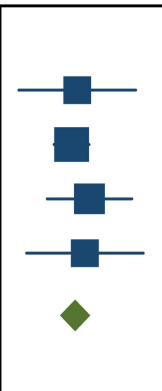

$2.94\left[\begin{array}{lll}1.30, & 6.62\end{array}\right] 6.61$

$2.71[2.13,3.46] \quad 74.17$

$3.48[1.94,6.27] 12.60$

$3.27\left[\begin{array}{lll}1.45, & 7.35] & 6.62\end{array}\right.$

$2.85[2.31,3.51]$

Figure 3 (A) Meta-analysis of associations between Cortical/medullary location and functional prognosis. (B) Meta-analysis of associations between PVS-SWI and early neurological deterioration/hemorrhagic transformation.

Abbreviations: PVS, prominent vessel sign; SWI, susceptibility-weighted imaging; End, unfavorable outcome; N-End, without unfavorable outcome; *mRS, scores 2-6 was regarded as unfavorable functional outcome. 
oxygenated hemoglobin ratio. This is consistent with our findings that the key determinants of PVS-SWI after an acute ischemic stroke are severe occlusions of the larger cerebral arteries/occlusion. Severe stenosis/occlusion of large arteries and inadequate oxygen supply result in compensatory dilated veins and larger penumbra volumes, ${ }^{31-33}$ causing early neurological deficits and ultimately leading to poor prognostic outcomes. This is similar to the findings of previous studies in which PVS-SWI was associated with poor prognosis in patients with ischemic stroke. ${ }^{10-20}$

\section{Modality of Treatment and Functional Prognosis}

According to the subgroup analysis related to the modality of treatment, there was a significant association between a poor prognosis and PVS on SWI in patients receiving thrombolytic therapy. An effective perfusion increases the availability of thrombolytic drugs and improves their ability to break blood clots. ${ }^{34}$ On the other hand, the presence of PVS-SWI indirectly reflects a severe hypoperfusion of brain tissues, resulting in a reduced availability of thrombolytic drugs and an increased risk of poor prognosis. ${ }^{13}$ Noteworthy, it is not thrombolysis per se that leads to poor prognosis, but that thrombolysis increases the difference in poor prognosis between the two groups of people with and without PVS-SWI. More than $80 \%$ of patients with PVSSWI have a poor prognosis if they are not treated with reperfusion therapy, whereas the rate decreases to $42 \%$ if they are treated with reperfusion. ${ }^{15}$ Animal models show that within minutes of an acute decrease in cerebral perfusion, $^{35}$ microthrombi are formed in intracranial small veins, leading to a reduced venous blood flow and an increased intracranial pressure, which further decreases cerebral perfusion and enlarges core infarcts, ${ }^{36,37}$ a process that may be blocked by an effective reperfusion therapy. In 10 patients with PVS who underwent SWI examination before and after recanalization, PVS-SWI disappeared in all patients after recanalization. ${ }^{38}$ If PVS-SWI is still present after treatment, the prognosis is poor. ${ }^{26}$ PVS-SWI can be a valid marker of acute ischemic stroke progression $^{32,39-42}$ A timely assessment of the ischemic penumbra and a successful reperfusion therapy are critical to avoid irreversible brain damage. ${ }^{43}$ Meanwhile, this present study showed that the risk of hemorrhagic transformation after stroke was independent of the presence of PVS-
SWI. Therefore, thrombolysis should not be delayed due to the presence of PVS-SWI.

Regarding the conservative treatment group, there was no significant association between PVS-SWI and a poor prognosis. We hypothesize that the following mechanisms explain the prognostic difference between patients with and without PVS-SWI: 1) The over-windowing and the presence of contraindications are probably the main reasons for not performing thrombolysis. This population has more underlying diseases and a worse overall prognosis than the thrombolytic group. 2) Patients with large infarcts and high cranial pressure have a deflated venous lumen, collapsed venous structures and reduced PVS-SWI visualization. ${ }^{44,45}$ These two mechanisms may have narrowed the difference in poor prognosis between the two groups of people with and without PVS-SWI.

\section{Cortical and Medullary PVS-SWI}

According to the subgroup analysis related to the location, both cortical and medullary veins were related to a poor prognosis. Compared to the cortex, medullary veins were a stronger predictor of poor prognosis. The deep medullary veins originate from the subcortical regions ${ }^{46,47}$ and play an important role in motor control and motor learning, functions having a strong impact on MRS scores. ${ }^{48-50}$ According to previous studies, patients with both cortical and medullary PVS had higher NIHSS scores and larger infarct sizes compared to patients with cortical PVS alone. ${ }^{16}$ It is possible that patients with medullary PVS have a greater need for thrombolytic therapy.

\section{Limitations}

This is the first study aimed to synthesize the relationship between PVS-SWI and functional outcomes, early neurological damage, and hemorrhagic transformation after an acute ischemic stroke. The large sample size, along with subgroup and sensitivity analyses ensured the soundness of our conclusions. However, our study has some limitations. First, the included studies are observational studies, which can cause a selection bias. Second, a high degree of heterogeneity was observed in the meta-analysis. Third, the small sample size related to the early neurological deterioration and hemorrhagic transformation may affect our conclusions. Fourth, the number of articles related to Interventional treatment and PVS-SWI was low and none of them met the inclusion criteria. 


\section{Conclusion}

In conclusion, PVS on SWI were related with a poor functional prognosis in patients treated with thrombolysis. Although the aggressive reperfusion therapy may be less effective in the PVS population, it may reduce the poor functional prognosis. We recommend routine PVS-SWI evaluation in patients with acute ischemic stroke to improve the prognosis of stroke. Large-scale trials are still needed to strengthen our understanding of PVS in ischemic stroke.

\section{Data Sharing Statement}

All data relevant to the study are included in the article or uploaded as supplementary information.

\section{Ethics Statement}

Not required.

\section{Acknowledgments}

We are grateful to all the articles and authors associated with the PVS-SWI.

\section{Author Contributions}

All authors made a significant contribution to the work reported, whether that is in the conception, study design, execution, acquisition of data, analysis and interpretation, or in all these areas; took part in drafting, revising or critically reviewing the article; gave final approval of the version to be published; have agreed on the journal to which the article has been submitted; and agree to be accountable for all aspects of the work.

\section{Funding}

This study is supported by 1) CAMS Innovation Fund for Medical Sciences (2019-I2M-5-029). 2) Beijing Municipal Committee of Science and Technology (Z20110000 5620010).

\section{Disclosure}

The research was conducted in the absence of any commercial or financial relationships that could be construed as a potential conflict of interest.

\section{References}

1. Knudsen AK, Collaborators GN. Disease burden in the Nordic region. Results from the global burden of disease study 2017 (GBD 2017). Eur J Public Health. 2019;29:65. doi:10.1093/eurpub/ckz185.161
2. Wang YJ, Li ZX, Gu HQ, et al. China stroke statistics 2019: a report from the National Center for Healthcare Quality Management in Neurological Diseases, China National Clinical Research Center for Neurological Diseases, the Chinese Stroke Association, National Center for Chronic and Non-communicable Disease Control and Prevention, Chinese Center for Disease Control and Prevention and Institute for Global Neuroscience and Stroke Collaborations. Stroke Vasc Neurol. 2020;5(3):211-239.

3. Chao BH, Yan F, Hua Y, et al. Stroke prevention and control system in China: CSPPC-stroke program. Int j Stroke. 2021;16(3):265-272. doi:10.1177/1747493020913557

4. Virani SS, Alonso A, Benjamin EJ, et al. Heart disease and stroke statistics-2020 update: a report from the American Heart Association. Circulation. 2020;141(9):e139-e596.

5. Haacke EM, Mittal S, Wu Z, Neelavalli J, Cheng YC. Susceptibilityweighted imaging: technical aspects and clinical applications, part 1. AJNR Am J Neuroradiol. 2009;30(1):19-30. doi:10.3174/ajnr.A1400

6. Mittal P, Kalia V, Dua S. Pictorial essay: susceptibility-weighted imaging in cerebral ischemia. Indian J Radiol Imaging. 2010;20 (4):250-253. doi:10.4103/0971-3026.73530

7. Jensen-Kondering U, Bohm R. Asymmetrically hypointense veins on $\mathrm{T} 2 * \mathrm{~W}$ imaging and susceptibility-weighted imaging in ischemic stroke. World J Radiol. 2013;5(4):156-165. doi:10.4329/wjr.v5. i4.156

8. Rauscher A, Sedlacik J, Barth M, Haacke EM, Reichenbach JR. Nonnvasive assessment of vascular architecture and function during modulated blood oxygenation using susceptibility weighted magnetic resonance imaging. Magn Reson Med. 2005;54(1):87-95. doi: $10.1002 / \mathrm{mrm} .20520$

9. Rosso C, Belleville M, Pires C, et al. Clinical usefulness of the visibility of the transcerebral veins at $3 \mathrm{~T}$ on $\mathrm{T} 2 *$-weighted sequence in acute stroke patients. Eur $J$ Radiol. 2012;81(6):1282-1287. doi:10.1016/j.ejrad.2011.03.025

10. Sun W, Liu W, Zhang Z, et al. Asymmetrical cortical vessel sign on susceptibility-weighted imaging: a novel imaging marker for early neurological deterioration and unfavorable prognosis. Eur J Neurol. 2014;21(11):1411-1418. doi:10.1111/ene. 12510

11. Yu J, Wang L, Li Z, Wang S, Wang G. Related factors of asymmetrical vein sign in acute middle cerebral artery stroke and correlation with clinical outcome. J Stroke Cerebrovasc Dis. 2017;26 (10):2346-2353. doi:10.1016/j.jstrokecerebrovasdis.2017.05.023

12. Wang Y, Shi T, Chen B, Lin G, Xu Y, Geng Y. Prominent hypointense vessel sign on susceptibility-weighted imaging is associated with clinical outcome in acute ischaemic stroke. Eur Neurol. 2018;79(56):231-239. doi:10.1159/000488587

13. Zhao G, Sun L, Wang Z, et al. Evaluation of the role of susceptibility-weighted imaging in thrombolytic therapy for acute ischemic stroke. J Clin Neurosci. 2017;40:175-179. doi:10.1016/j. jocn.2017.01.001

14. Yu X, Yuan L, Jackson A, et al. Prominence of medullary veins on susceptibility-weighted images provides prognostic information in patients with subacute stroke. Am $J$ Neuroradiol. 2016;37 (3):423-429. doi:10.3174/ajnr.A4541

15. Zhang X, Zhang S, Chen Q, Ding W, Campbell BCV, Lou M. Ipsilateral prominent thalamostriate vein on susceptibility-weighted imaging predicts poor outcome after intravenous thrombolysis in acute ischemic stroke. AJNR Am $J$ Neuroradiol. 2017;38 (5):875-881. doi:10.3174/ajnr.A5135

16. Jing L, Sui B, Shen M, Qin H, Gao P. Are prominent medullary veins better than prominent cortical veins as predictors of early clinical outcome in patients with acute ischemic stroke? Diagn Interventional Radiol. 2021;27(2):285-292.

17. Vural A, Gocmen R, Oguz KK, Topcuoglu MA, Arsava EM. Bright and dark vessels on stroke imaging: different sides of the same coin? Diagn Interventional Radiol. 2016;22(3):284-290. doi:10.5152/ dir.2015.15271 
18. Li W, Xiao WM, Luo GP, et al. Asymmetrical cortical vein sign predicts early neurological deterioration in acute ischemic stroke patients with severe intracranial arterial stenosis or occlusion. $B M C$ Neurol. 2020;20(1). doi:10.1186/s12883-020-01907-w.

19. Liu YL, Yin HP, Qiu DH, et al. Multiple hypointense vessels on susceptibility-weighted imaging predict early neurological deterioration in acute ischaemic stroke patients with severe intracranial large artery stenosis or occlusion receiving intravenous thrombolysis. Stroke Vasc Neurol. 2020;5(4):361-367. doi:10.1136/svn-2020000343

20. Zongji H, Qi T, Lin L, et al. Correlation between asymmetrically prominent cortical veins on susceptibility-weighted imaging and early neurological deterioration in patients with acute ischemic stroke. Int J Cerebrovasc Dis. 2020;28(2):87-92.

21. Liu H, Mei W, Huang Y, Li Y, Liu H, Zhang J. The association between asymmetrically hypointense veins on susceptibility-weighted imaging and collateral circulation. Chin $J$ Neurol. 2018;51(1):21-27.

22. Chen CY, Chen CI, Tsai FY, Tsai PH, Chan WP. Prominent vessel sign on susceptibility-weighted imaging in acute stroke: prediction of infarct growth and clinical outcome. PLoS One. 2015;10(6): e0131118.

23. Ya-Nan J, Cui-Cui L, Jun-Yan L.Predictive value of asymmetrical cortical vein sign based on susceptibility weighted imaging for prognosis of patients with acute ischemic stroke. Chin J Stroke. 2019;14 (7):639-644.

24. Wang $\mathrm{C}, \mathrm{Wu} \mathrm{S}$, Gong X. Asymmetrical hypointense cortical veins on susceptibility weighted imaging in patients with acute cerebral infarction. Chin J Neurol. 2017;50(6):452-456.

25. Huang P, Chen CH, Lin WC, Lin RT, Khor GT, Liu CK. Clinical applications of susceptibility weighted imaging in patients with major stroke. J Neurol. 2012;259(7):1426-1432. doi:10.1007/s00415-0116369-2

26. Moher D, Liberati A, Tetzlaff J, Altman DG, Group P. Preferred reporting items for systematic reviews and meta-analyses: the PRISMA statement. J Clin Epidemiol. 2009;62(10):1006-1012. doi:10.1016/j.jclinepi.2009.06.005

27. Liu YL, Xiao WM, Lu JK, et al. Asymmetrical cortical vessel sign predicts prognosis after acute ischemic stroke. Brain Behav. 2020;10 (7). doi: $10.1002 / \mathrm{brb3} .1657$.

28. Young AR, Sette G, Touzani O, et al. Relationships between high oxygen extraction fraction in the acute stage and final infarction in reversible middle cerebral artery occlusion: an investigation in anesthetized baboons with positron emission tomography. $J$ Cereb Blood Flow Metab. 1996;16(6):1176-1188. doi:10.1097/00004647199611000-00012

29. Kennan RP, Zhong J, Gore JC. Intravascular susceptibility contrast mechanisms in tissues. Magn Reson Med. 1994;31(1):9-21. doi:10.1002/mrm.1910310103

30. Xu Z, Duan Y, Yang B, Huang X, Pei Y, Li X. Asymmetric deep medullary veins in patients with occlusion of a large cerebral artery: association with cortical veins, leptomeningeal collaterals, and prognosis. Front Neurol. 2019;10:1292. doi:10.3389/fneur.2019.01292

31. Fujioka M, Okuchi K, Iwamura A, Taoka T, Siesjo BK, Mismatch A. Between the abnormalities in diffusion- and susceptibility-weighted magnetic resonance imaging may represent an acute ischemic penumbra with misery perfusion. J Stroke Cerebrovasc. 2013;22 (8):1428-1431.

32. Luo S, Yang L, Wang L. Comparison of susceptibility-weighted and perfusion-weighted magnetic resonance imaging in the detection of penumbra in acute ischemic stroke. $J$ Neuroradiol. 2015;42 (5):255-260. doi:10.1016/j.neurad.2014.07.002

33. Payabvash S, Taleb S, Benson JC, et al. Susceptibility-diffusion mismatch in middle cerebral artery territory acute ischemic stroke: clinical and imaging implications. Acta Radiol. 2017;58(7):876-882. doi: $10.1177 / 0284185116675658$
34. Alves HC, Treurniet KM, Dutra BG, et al. Associations between collateral status and thrombus characteristics and their impact in anterior circulation stroke. Stroke. 2018;49(2):391-396. doi:10.1161/STROKEAHA.117.019509

35. Zhou Y, Wang Y, Wang J, Anne Stetler R, Yang QW. Inflammation in intracerebral hemorrhage: from mechanisms to clinical translation. Prog Neurobiol. 2014;115:25-44. doi:10.1016/j.pneuro bio.2013.11.003

36. Schwarzmaier SM, Kim SW, Trabold R, Plesnila N. Temporal profile of thrombogenesis in the cerebral microcirculation after traumatic brain injury in mice. $J$ Neurotrauma. 2010;27(1):121-130. doi:10.1089/neu.2009.1114

37. Li Q, Khatibi N, Zhang JH. Vascular neural network: the importance of vein drainage in stroke. Transl Stroke Res. 2014;5(2):163-166. doi:10.1007/s12975-014-0335-0

38. Baik SK, Choi W, Oh SJ, et al. Change in cortical vessel signs on susceptibility-weighted images after full recanalization in hyperacute ischemic stroke. Cerebrovasc Dis. 2012;34(3):206-212. doi:10.1159/ 000342148

39. Wang XC, Gao PY, Xue J, Liu GR, Ma L. Identification of infarct core and penumbra in acute stroke using CT perfusion source images. AJNR Am J Neuroradiol. 2010;31(1):34-39. doi:10.3174/ajnr.A1740

40. Meoded A, Poretti A, Benson JE, Tekes A, Huisman TA. Evaluation of the ischemic penumbra focusing on the venous drainage: the role of susceptibility weighted imaging (SWI) in pediatric ischemic cerebral stroke. J Neuroradiol. 2014;41(2):108-116. doi:10.1016/j. neurad.2013.04.002

41. Fujioka M, Okuchi K, Iwamura A, Taoka T, Siesjö BK. A mismatch between the abnormalities in diffusion- and susceptibility-weighted magnetic resonance imaging may represent an acute ischemic penumbra with misery perfusion. J Stroke Cerebrovasc Dis. 2013;22 (8):1428-1431. doi:10.1016/j.jstrokecerebrovasdis.2012.12.009

42. Fisher M. The ischemic penumbra: identification, evolution and treatment concepts. Cerebrovasc Dis. 2004;17(Suppl 1):1-6. doi:10.1159/ 000074790

43. Darwish EAF, Abdelhameed-El-Nouby M, Geneidy E. Mapping the ischemic penumbra and predicting stroke progression in acute ischemic stroke: the overlooked role of susceptibility weighted imaging. Insights Imaging. 2020;11(1):6. doi:10.1186/s13244-0190810-y

44. Viallon M, Altrichter S, Pereira VM, et al. Combined use of pulsed arterial spin-labeling and susceptibility-weighted imaging in stroke at 3T. Eur Neurol. 2010;64(5):286-296. doi:10.1159/000321162

45. $\mathrm{Xia} \mathrm{H}$, Sun $\mathrm{H}, \mathrm{He} \mathrm{S}$, et al. Absent cortical venous filling is associated with aggravated brain edema in acute ischemic stroke. AJNR Am $J$ Neuroradiol. 2021;42(6):1023-1029. doi:10.3174/ajnr.A7039

46. Oran I, Memis A, Sener RN, Yunten N. The so-called transcerebral veins: appearance in three different cases. Comput Med Imaging Graphics. 1999;23(3):127-131. doi:10.1016/S0895-6111(99)00009-9

47. Friedman DP. Abnormalities of the deep medullary white matter veins: MR imaging findings. AJR Am J Roentgenol. 1997;168 (4):1103-1108. doi:10.2214/ajr.168.4.9124123

48. Wichmann T, DeLong MR. Functional and pathophysiological models of the basal ganglia. Curr Opin Neurobiol. 1996;6(6):751-758. doi:10.1016/S0959-4388(96)80024-9

49. Mink JW, Thach WT. Basal ganglia motor control. III. Pallidal ablation: normal reaction time, muscle cocontraction, and slow movement. $J$ Neurophysiol. 1991;65(2):330-351. doi:10.1152/ jn.1991.65.2.330

50. DeLong MR, Alexander GE, Mitchell SJ, Richardson RT. The contribution of basal ganglia to limb control. Prog Brain Res. 1986;64:161-174. 


\section{Publish your work in this journal}

Neuropsychiatric Disease and Treatment is an international, peerreviewed journal of clinical therapeutics and pharmacology focusing on concise rapid reporting of clinical or pre-clinical studies on a range of neuropsychiatric and neurological disorders. This journal is indexed on PubMed Central, the 'PsycINFO' database and CAS, and is the official journal of The International Neuropsychiatric Association (INA). The manuscript management system is completely online and includes a very quick and fair peer-review system, which is all easy to use. Visit http://www.dovepress.com/testimonials.php to read real quotes from published authors.

Submit your manuscript here: https://www.dovepress.com/neuropsychiatric-disease-and-treatment-journal 